\section{Early use of abiraterone and radium-223 in metastatic prostate} cancer

Matthew Smith and colleagues ${ }^{1}$ presented the results of the phase 3 ERA 223 trial, in which abiraterone plus prednisone were combined with radium-223 or placebo to treat patients with chemotherapy-naive castration-resistant prostate cancer with bone metastases. Against all expectations, the study failed in its primary endpoint: the addition of radium-223 to abiraterone plus prednisone did not improve symptomatic skeletal events-free survival, but was associated with increased risk fracture, mainly in nonmetastatic bone sites. The editorial article by Daniel Spratt ${ }^{2}$ suggested that the increased bone fragility could be due to the synergistic inhibitory effect on osteoblasts from abiraterone and prednisone, combined with the osteoclastogenic effects of androgen deprivation, glucocorticoids, and radium-223. In this regard, we have some concerns.

Another possible reason that could explain the results of the ERA 223 study is the anabolic and antiresorptive effect of abiraterone on the bone. Indeed, our previous evidence showed that abiraterone treatment inhibited osteoclastic function, and promoted osteoblastic differentiation and bone matrix deposition, modulating the gene expression of these cells. ${ }^{3}$ Since radium-223 deposits mostly in active bone remodelling areas with high osteoblastic activity, the combination with abiraterone could induce its accumulation not only at metastatic sites, but also in healthy bone. Therefore, this abnormal deposition of radium-223 in healthy bone and the subsequent $\alpha$-particle emission could lead to cell and tissue damage. This noteworthy effect of abiraterone in healthy bone has not been observed in our study with enzalutamide (unpublished), which could contribute to the lack of alert to bone fractures in the ongoing Peace 3 trial (NCT02194842) treating patients with radium-223 combined with enzalutamide.

Contrary to ERA 223 data, previous clinical studies ${ }^{4.5}$ have shown positive results of the abiraterone and radium-223 combination, in terms of safety and efficacy. In these studies, the majority of enrolled patients were in the post-docetaxel setting; therefore, it is plausible that a previous cytotoxic therapy could reduce the anabolic response of healthy bone to the subsequent abiraterone treatment.

Smith and colleagues suggest the use of drugs for bone health to reduce skeletal morbidity with concomitant use of abiraterone and radium-223. However, in our opinion, the anabolic and antiresorptive effect of abiraterone warrants caution when these treatments are combined in the early settings of the disease, such as in the ERA 223 trial. The combination of new-generation drugs is developing within a largely unknown field that concerns the multifactorial mechanisms of bone stimulation; in this scenario, translational medicine could contribute to solve unanswered questions.

UD reports personal fees from Janssen, Astellas, Sanofi, and Bayer. MCC, DS, MI, and GP declare no competing interests.

*Maria Concetta Cursano, Daniele Santini, Michele Iuliani, Giovanni Paganelli, Ugo De Giorgi m.cursano@unicampus.it

Department of Medical Oncology, Campus Bio-Medico University of Rome, 00128 Rome, Italy (MCC, DS, MI); and Department of Medical Oncology (UD), and Nuclear Medicine Unit (GP), Istituto Scientifico Romagnolo per lo Studio e la Cura dei Tumori, Meldola, Italy

1 Smith M, Parker C, Saad F, et al. Addition of radium-223 to abiraterone acetate and prednisone or prednisolone in patients with castration-resistant prostate cancer and bone metastases (ERA 223): a randomised, double-blind, placebo-controlled, phase 3 trial. Lancet Oncol 2019; 20: 408-19.

2 Spratt DE. Combination therapies in prostate cancer: proceed with caution. Lancet Oncol 2019; 20: 321-23.
3 Iuliani M, Pantano F, Buttigliero C, et al. Biological and clinical effects of abiraterone on anti-resorptive and anabolic activity in bone microenvironment. Oncotarget 2015; 6: 12520-28.

4 Saad F, Carles J, Gillessen S, et al. Radium-223 and concomitant therapies in patients with metastatic castration-resistant prostate cancer: an international, early access, open-label, single-arm phase $3 \mathrm{~b}$ trial. Lancet Oncol 2016; 17: 1306-01

5 Sartor O, Vogelzang NJ, Sweeney C, et al Radium-223 safety, efficacy, and concurrent use with abiraterone or enzalutamide: first US experience from an expanded access program. Oncologist 2018; 23: 193-202. 